\title{
L'enseignement espagnol à Paris sous la Restauration et la monarchie de Juillet
}

Spanish education in Paris under the Restoration and the July Monarchy

Anne Leblay-Kinoshita

\section{OpenEdition}

Journals

Édition électronique

URL : https://journals.openedition.org/histoire-education/2702

DOI : $10.4000 /$ histoire-education.2702

ISSN : 2102-5452

Éditeur

ENS Éditions

Édition imprimée

Date de publication : 20 décembre 2013

Pagination : $5-29$

ISBN : 978-2-84788-502-6

ISSN : 0221-6280

Référence électronique

Anne Leblay-Kinoshita, «L'enseignement espagnol à Paris sous la Restauration et la monarchie de Juillet », Histoire de l'éducation [En ligne], 139 | 2013, mis en ligne le 15 décembre 2015, consulté le 20 mai 2021. URL : http://journals.openedition.org/histoire-education/2702 ; DOI : https://doi.org/ 10.4000/histoire-education.2702 


\title{
L'enseignement espagnol à Paris sous la Restauration et la monarchie de Juillet
}

\author{
Anne Leblay-Kinoshita
}

Sous la Restauration, trois établissements scolaires espagnols ouvrent leurs portes à Paris. En 1816, l'abbé Gregorio Alonso de Prado fonde une pension rue de la Michodière. En 1827, Manuel Silvela García de Aragón, ancien professeur de droit, transfère rue de Montreuil un collège espagnol, créé onze ans plus tôt à Bordeaux. En 1828, Domingo Fernández Ángulo, négociant, ouvre à son tour une institution rue de Beautreillis. Quelques années après leur création, les trois établissements disparaissent brutalement.

La période où ils surgissent correspond à l'arrivée de nombreux réfugiés espagnols en France. Entre la fin du Premier Empire et la monarchie de Juillet, des dizaines de milliers d'Espagnols trouvent asile au nord des Pyrénées : des afrancesados, partisans de Joseph Bonaparte, lors de la retraite des Français en 1813, des absolutistes pendant le Trienio liberal, des libéraux jusqu'à la mort de Ferdinand VII en 1833, des ultraroyalistes comme les agraviados en 1828 et 1829. Pendant la régence de Marie-Christine de Bourbon, c'est le tour des carlistes, dont le nombre atteint près de 40000 personnes vers $1840^{1}$. Des réfugiés continuent d'arriver, en nombre plus modéré, jusqu'à la fin de la monarchie de Juillet : des christinos, partisans de l'ancienne régente exilée entre 1840 et 1844 , des "espartéristes", partisans de Baldomero Espartero, régent entre 1841 et 1843, ou d'autres émigrés de différentes tendances progressistes.

La présence d'émigrés ${ }^{2}$ espagnols en France est une constante dans la

1 Emmanuel Tronco, Les Carlistes espagnols dans l'Ouest de la France, Rennes, Presses universitaires de Rennes, 2010, p. 179.

2 Dans cet article, nous utilisons les termes de "réfugié" ou d'" émigré». Si la connotation politique du premier est communément admise (bien qu'elle tende aujourd'hui à s'élargir puisque l'on parle 
première moitié du XIX ${ }^{\mathrm{e}}$ siècle. À Paris, leur nombre est également important : 200 chefs de famille entre 1815 et $1830^{3}, 1350$ personnes en $1833^{4}$ avant que leur nombre ne redescende autour d'un demi-millier en 1836, après plusieurs campagnes d'amnistie ${ }^{5}$. Les autorités françaises tentent pourtant de limiter leur présence dans la capitale. À la fin du Premier Empire, seuls les réfugiés de marque, non secourus, sont autorisés à y séjourner ${ }^{6}$. Au début de la Restauration, les Espagnols qui séjournent à Paris sont éloignés par le ministère de la Guerre et assignés à résidence dans différentes villes du sud de la France ${ }^{7}$. Ce principe perdure jusqu'à la loi du 21 avril 1832 qui définit un statut pour les réfugiés étrangers. Cette politique est assortie de différentes mesures d'expulsion de Paris des réfugiés italiens, espagnols, portugais puis polonais entre décembre 1830 et juin $1832^{8}$. Les autorités souhaitent éviter le regroupement de personnalités étrangères aux activités politiques jugées subversives ou susceptibles de porter atteinte aux bonnes relations diplomatiques de la France avec les États voisins. Ce principe de limitation est en général fondé sur un critère financier : à moins qu'ils ne portent atteinte à l'ordre public, seuls les réfugiés secourus par l'État sont réellement soumis à une résidence obligée ${ }^{9}$. Depuis la fin du Premier Empire et jusqu'à la monarchie de Juillet, les réfugiés espagnols bénéficient en effet, avec quelques interruptions selon le régime politique, les idées qu'ils défendent ou leur situation personnelle, d'un "subside", secours grossièrement défini en fonction de la classe sociale d'origine ${ }^{10}$.

L'attraction parisienne s'explique notamment par la centralisation à Paris

désormais de "réfugiés climatiques»), l'usage du second peut poser question. Nous pouvons d'abord considérer le terme "émigré" de façon neutre, dans son sens étymologique, qui désigne une personne allant d'un lieu à un autre, de façon durable, sans précisions sur ses motivations. Toutefois, au début du XIX ${ }^{e}$ siècle, dans le contexte postrévolutionnaire français, ce terme possède une forte connotation politique. Le terme "exilé" est alors davantage utilisé dans le champ littéraire.

3 Anne Leblay, Les réfugiés ibériques libéraux à Paris au temps des monarchies constitutionnelles, thèse de doctorat, École des hautes études en sciences sociales, 2013, p.72, 76 et 79.

4 Paul Gerbod, "À propos des étrangers en France sous la monarchie de Juillet", Annales de démographie historique, 1987, p. 346.

5 Anne Leblay, Les réfugiés ibériques libéraux à Paris..., op. cit., p. 78-82.

6 Juan López Tabar, Los famosos traidores, los afrancesados durante la crisis del Antiguo Régimen, 1808-1833, Madrid, Biblioteca Nueva, 2001, p. 111.

7 Archives nationales [désormais AN], F/7/12109, notes et affaires diverses. Guerre. Ministre de la Guerre au ministre de la Police générale, Paris, le 26 juillet 1815. Extrait.

8 AN, F/7/12102, renvoi des réfugiés de Paris. Préfet de police au ministre de l'Intérieur, Paris, le 21 juin 1832 .

9 Anne Leblay, Les réfugiés ibériques libéraux à Paris..., op. cit., p. 171, 175, 183-190.

10 Ibid., p. 155-198. 
du pouvoir politique, d'activités économiques et financières dynamiques, et de la plupart des institutions culturelles ou savantes. Ainsi, en 1831, 14\% des réfugiés espagnols exercent une activité comme professeur, artiste, traducteur ou interprète, sans compter quelques journalistes, et $10 \%$ des réfugiés espagnols de la capitale suivent des études ${ }^{11}$, notamment dans le domaine médical. De 1807 à 1830, dix-neuf Espagnols, réfugiés ou non, reçoivent leur doctorat à l'École de médecine, parmi 294 étudiants ${ }^{12}$. Enfin, pour quelques-uns d'entre eux, cet investissement dans la sphère éducative prend une autre dimension, puisque certains fondent les établissements d'éducation cités plus haut.

Il s'agit donc de comprendre s'il existe des pratiques éducatives spécifiques aux Espagnols. Quelle est la part de volontarisme ou de nécessité dans la diffusion de leur langue? Que transmettent-ils de leur culture originelle et de ce qu'elle représente aux yeux de leurs compatriotes comme des étrangers. Comment se positionnent-ils par exemple par rapport à la question de la place de la religion, de l'idée romantique d'une certaine décadence espagnole (avec la perte des colonies espagnoles notamment)? Gue retiennent-ils des principes humanistes diffusés par la Révolution française qu'ils (re)découvrent en arrivant en France? Des réponses sont esquissées, des hypothèses sont émises, même si les silences restent nombreux en raison de la rareté des témoignages et de la modestie des sources d'archives publiques à l'égard d'une population qui n'intéresse alors guère les autorités.

\section{I - L'importance des réfugiés espagnols dans la diffusion de la langue espagnole à Paris}

La majorité des réfugiés espagnols qui séjournent à Paris, même ceux issus de milieux favorisés ou bénéficiant du subside de l'État, doivent trouver de nouvelles sources de revenus. La langue maternelle peut devenir une ressource à une époque où peu de Français maîtrisent une langue vivante étrangère. Les cours de langue et la traduction constituent des activités d'appoint faciles à mettre en œuvre : même si les revenus procurés sont modestes, elles n'exigent pas ou quasiment pas de mise de fonds initiale. L'édition en langue espagnole est

\footnotetext{
11 Ibid., p. 512.

12 Pierre Moulinier, Les étudiants étrangers à Paris au XIX ${ }^{e}$ siècle. Migration et formation des élites, Rennes, Presses universitaires de Rennes, 2012, p. 48.
} 
alors une activité en pleine croissance, ce qui permet en amont le recrutement de traducteurs et d'ouvriers dans le secteur de l'imprimerie, en particulier au sein des réfugiés espagnols ${ }^{13}$. De 1814 à 1833, 893 ouvrages en espagnol sont publiés en France, avec une moyenne par tirage de 1100 exemplaires (la moyenne est alors d'environ 500 exemplaires). Au sein de cette production, les manuels de castillan occupent une place considérable avec environ 120 titres, soit plus de six manuels par an entre 1814 et 1833. Les ouvrages espagnols arrivent au troisième rang des publications en langue étrangère, après celles en anglais et en allemand ${ }^{14}$. Cette croissance s'explique par la présence d'une clientèle espagnole, l'ouverture du marché sud-américain après l'émancipation des anciennes colonies espagnoles ainsi que la modestie du secteur éditorial dans la péninsule ibérique, pour des raisons économiques et politiques, ce qui encourage un commerce de contrebande depuis le royaume français. La production de manuels scolaires est particulièrement abondante entre 1823 et 1826 et entre 1838 et 1846, avec des pics en 1842 et $1845^{15}$. Même s'il convient de rester prudent dans l'interprétation de ces chiffres modestes, il semble exister une simultanéité avec les vagues d'exil espagnol. Le premier pic correspond à l'arrivée de réfugiés libéraux après l'intervention française en Espagne et la reprise du pouvoir par Ferdinand VII. Le second correspond à l'arrivée des réfugiés carlistes et à la période d'exil de la régente Marie-Christine et de ses partisans.

Les manuels d'espagnol peuvent s'adresser aux enfants des expatriés. En 1825, les Espagnols de moins de vingt-cinq ans représentent en effet près de $40 \%$ de l'effectif réfugié à Paris ${ }^{16}$. Ils paraissent toutefois surtout viser le marché hispanophone, en Espagne ou en Amérique du Sud. Ainsi, l'expression de "traductor para América", apparemment courante parmi les réfugiés, illustre l'importance de ce phénomène ${ }^{17}$. Le réfugié Vicente Salvá s'associe en juin 1830 à l'imprimeur-libraire français Bossange pour fonder une nouvelle

13 Anne Leblay, Les réfugiés ibériques libéraux à Paris..., op. cit., p. 521-522.

14 Aline Vauchelle Haquet, Les ouvrages en langue espagnole publiés entre 1814 et 1833, Aix-enProvence, Publications de l'université de Provence, 1985, p.33-37.

15 Chiffres obtenus à partir de la liste des ouvrages recensés dans AlainChoppin, FabiolaRodriguez, BertrandPinhède, Manuels scolaires en France de 1789 à nos jours, vol. 6. Les manuels d'espagnol, Paris, INRP, 1995.

16 Anne Leblay, Les réfugiés ibériques libéraux à Paris..., op. cit., p. 481 et 484.

17 Vicente Lloréns Castillo, Liberales y románticos. Una emigración española en Inglaterra (1823-1834), México, Nueva revista de filología hispánica, 1954, p. 129. 
maison d'édition au nom significatif : la Librairie hispano-américaine ${ }^{18}$. De façon beaucoup plus modeste, ces ouvrages répondent également au besoin ou au souhait de quelques Français d'apprendre les rudiments de la langue castillane pour communiquer avec les nouveaux arrivants. La plupart des fonctionnaires en charge des réfugiés au ministère de l'Intérieur ne connaissent en effet pas les langues étrangères, ce qui ne facilite pas le traitement des dossiers. Les confusions entre les langues méridionales ne sont pas rares à une époque où viennent trouver refuge simultanément les ressortissants des péninsules ibérique et italienne. Le ministre de l'Intérieur invite ainsi les préfets à faire preuve de vigilance en raison de "l'analogie que présentent entre elles les langues des pays méridionaux de l'Europe ${ }^{19}$. Ces publications font aussi probablement écho à une mode espagnole qui touche un large public parisien. Dans la première moitié du XIX" siècle, c'est "le chemin de la culture espagnole dans son ensemble que retrouve alors la France ${ }^{20}$ : la guitare devient populaire avec Fernando Sor, "afrancesado" établi à Paris depuis $1813^{21}$, Maria Malibran, cantatrice d'origine espagnole, est au sommet de sa gloire à Paris, Prosper Mérimée encense l'Espagne dans son œuvre, depuis le Théâtre de Clara Gazul (1825) jusqu'à l'Histoire de don Pèdre Ir, roi de Castille (1847) ${ }^{22}$. L'occupation de l'Espagne par des troupes françaises entre 1809 et 1813 puis de façon plus limitée entre 1823 et 1827 et le grand nombre de réfugiés espagnols présents en France participent vraisemblablement de cette vogue du goût espagnol. Les langues vivantes ont alors une place réduite dans l'enseignement secondaire français. Leur apprentissage ne devient obligatoire qu'en 1838. Il s'agit alors d'une heure de cours par semaine, selon des méthodes inspirées de l'enseignement des langues anciennes, et généralement dispensée par un personnel médiocre. Négligé voire méprisé, le castillan demeure en outre peu enseigné car il jouit, avec l'italien, d'une réputation de trop grande facilité par rapport

18 Carola Reig Salvá, Vicente Salvá, un Valenciano de prestigio internacional, Valence, Instituto de literatura y estudios filológicos, Institución Alfonso el Magnánimo, Diputación provincial de Valencia, CSIC, 1972, p. 123.

19 AN, F/la/40, circulaire du ministre de l'Intérieur, Paris, le 12 juin 1835.

20 Selon les termes de Jean-Claude Berchet dans l'introduction à : Théophile Gautier, Voyage en Espagne, Paris, Flammarion, 1981, p. 18.

21 Jean-René Aymes, Los Españoles en París en la época romántica, Madrid, Alianza editorial, 2008, p. 202-203.

22 En ligne : <http://www.merimee.culture.fr/fr/html/ec/ec_2_3.html> : "Espagne : la patrie d'élection" (consulté le 4 octobre 2014). 
à l'allemand et à l'anglais ${ }^{23}$. Entre 1842 et 1847, 43 candidats sont reçus au certificat d'aptitude à l'enseignement des langues vivantes en allemand, 48 en anglais, mais seulement 11 en italien et 9 en espagnol, quasiment la moitié d'entre eux étant vraisemblablement d'origine espagnole ${ }^{24}$.

L'importance de l'édition espagnole en France participe donc à la diffusion de la langue. Les frontières entre l'interprétariat, la traduction et l'enseignement ne sont pas faciles à tracer. L' "afrancesado" Manuel Núñez de Taboada, "directeur de l'interprétation générale des langues, établissement connu depuis 1811 ", propose à la fois des "traductions authentiques, commerciales, littéraires et judiciaires", le déchiffrement des "écritures les plus anciennes en quelque langue que ce soit" ainsi que des "cours publics et particuliers permanents de toutes les langues européennes et des principales langues orientales ${ }^{25}$. Dans l'Almanach du commerce de 1833, les interprètes assermentés sont classés dans la rubrique des professeurs de langue. Eugenio Biezma Guerrero œuvre "au tribunal de commerce, comme examinateur et membre du jury pour l'admission des élèves de la marine royale de France", dirige des "bureaux de traductions authentiques, commerciales, littéraires et judiciaires" et propose des "cours permanens et leçons particulières de langues espagnole et portugaise $»^{26}$. Les réussites de Núñez de Taboada et de Biezma Guerrero restent exceptionnelles, mais la pratique simultanée de la traduction et de l'enseignement parait courante. Quelques heures de cours par semaine ou quelques traductions payées à la pièce fournissent un revenu d'appoint : le réfugié Juan Vallarino gagne sa vie en rédigeant des articles sur l'Espagne pour des journaux parisiens, en traduisant "plusieurs ouvrages tels que les œuvres de Franklin et l'histoire de Bonaparte pour les envoyer en Amérique" et en donnant "en outre des leçons de langue espagnole à plusieurs Anglais; et il est tellement occupé qu'il ne veut recevoir personne ${ }^{27}$.

Pourtant seuls onze professeurs d'espagnol sont recensés dans l'Almanach du commerce de 1833, parmi lesquels quatre probablement d'origine espagnole.

23 Paul Gerbod, La condition universitaire en France, Paris, Presses universitaires de France, 1965, p. 76 et Alain Choppin et al., Manuels scolaires en France de 1789 à nos jours, op. cit., 1995, p.23.

24 Auguste Wolfromm (dir.), Annuaire de l'enseignement des langues vivantes. $1^{\text {re }}$ année, Paris, A. Laisney, Le Havre, L. Murer, 1891, p. 164-179. Figurent ainsi parmi les certifiés les dénommés Gallo de Cuendias, Escriche, De Mendia et Arana.

25 Almanach du commerce de Paris de 1837, Paris, Bureau de l'Almanach, 1837, p. 284.

26 Almanach du commerce de Paris de 1833, Paris, Bureau de l'Almanach, 1833, p. 255.

27 AN, F/7/11994, Vicente Canaviral. Antonio Barcia, Juan Vallerino ou Vallarino. Préfet de police au ministre de l'Intérieur, Paris, le 18 avril 1826. 
L'Agenda des pensions de 1836 mentionne six professeurs dont trois originaires de la péninsule ibérique ${ }^{28}$. Les "maitres de langue", enseignants souvent occasionnels, ne sont pas recensés dans ce genre d'ouvrage et ont même parfois mauvaise réputation : en 1831, le préfet de police de Paris demande l'expulsion de tous les réfugiés italiens "qui n'auraient pas 60 ans ou qui n'exerceraient pas une profession réelle, autre cependant que celles de maître de musique et d'italien. Professions qu'ils ne manqueraient pas d'invoquer tous "29. Il n'y a en effet aucun moyen d'évaluer les compétences de ces enseignants autoproclamés et, plus grave pour les autorités, leur statut précaire leur assure des revenus trop incertains.

C'est également une activité à laquelle les femmes peuvent prétendre. C'est même l'un des rares secteurs, dès lors qu'il s'agit d'éduquer des enfants ou des adolescents et non d'instruire des adultes, où leur intervention est considérée comme légitime. Elle est alors perçue comme une extension de la fonction naturelle de la mère au sein d'un foyer ${ }^{30}$. En outre, alors que les langues classiques restent le domaine réservé des hommes, les femmes de l'élite bourgeoise peuvent accéder à l'apprentissage et donc à l'enseignement des langues vivantes qui relèvent quasiment des bonnes manières. La grille d'inspection des pensionnats de jeunes filles à Paris comprend par exemple une rubrique intitulée "langues étrangères et arts d'agrément ${ }^{31}$. Cette possibilité d'exercer une activité s'avère précieuse pour les réfugiées seules. En effet, les Espagnoles non accompagnées ne peuvent alors prétendre au statut de réfugiées politiques et donc bénéficier du subside gouvernemental ${ }^{32}$. Madame Díaz de la Peña, veuve d'un bourgeois afrancesado de Salamanque, donne quelques cours d'espagnol et d'italien vers 1818, avant de trouver un revenu plus régulier comme éducatrice dans une famille anglaise de Sèvres $^{33}$.

28 Almanach du commerce de Paris de 1833, op. cit., p. 255-256 et Almanach du commerce de Paris de 1837, op. cit., p. 284-285; Légerson, Agenda des pensions, collèges, gymnases, externats, écoles, etc., dans Paris et les environs. Livre-souvenir de Poche, contenant tous les renseignements spéciaux utiles ou agréables aux parents, instituteurs et enfants, Paris, Bréauté, 1836.

29 AN, F/7/12102, renvoi des réfugiés de Paris. Préfet de police au ministre de l'Intérieur, Paris, le 10 août 1831, réfugiés italiens. Confidentielle.

30 Yannick Ripa, Les femmes, actrices de l'histoire, France, de 1789 à nos jours, Paris, Armand Colin, 2010, p. 39.

31 Rebecca Rogers, "Les femmes dans l'enseignement des langues vivantes : éléments pour une histoire à construire", Ela. Études de linguistique appliquée, nº 142, 2006, p. 137.

32 Anne Leblay, Les réfugiés ibériques libéraux à Paris..., op. cit., p. 192-193.

33 Jean-René Aymes, Los Españoles en París en la época romántica, op. cit., 2008, p. 243. 
Le profil des élèves recoupe vraisemblablement celui des utilisateurs de manuels de langue évoqués plus haut : ceux qui apprennent en raison de leurs activités professionnelles, ceux qui s'intéressent à la culture espagnole et, bien entendu, les enfants hispanophones.

\section{II - Les établissements espagnols à Paris au service de la «cause» des réfugiés?}

Sous la Restauration, trois établissements scolaires sont fondés à Paris par des Espagnols : en 1816, la pension de l'abbé Gregorio Alonso de Prado, reprise en 1828 par José Mariano Vallejo; en 1827, l'institution de Manuel Silvela García de Aragón, créée onze ans plus tôt à Bordeaux; et, en 1828, le collège de Domingo Fernández Ángulo. L'existence d'un collège dirigé par Vicente González Arnao, réfugié afrancesado, est également évoquée mais aucun établissement à son nom n'a été identifié dans les fonds de l'Instruction publique ${ }^{34}$. Ainsi, en décembre 1828, il existe officiellement au moins trois établissements privés espagnols à Paris ${ }^{35}$. Leur existence est limitée dans le temps. En 1836, l'Agenda des pensions n'en recense aucun ${ }^{36}$. Les établissements dirigés par Prado puis Vallejo, Silvela et Ángulo ont probablement disparu entre 1832 et 1834. Ils sont localisés rive droite dans l'ancien huitième arrondissement, de part et d'autre de la place de la Bastille. En s'éloignant du centre, le bâti est moins dense et il est probablement plus facile et moins coûteux de trouver des locaux pouvant accueillir des classes, des dortoirs et des espaces de récréation. L'abbé Prado et Fernández Ángulo trouvent des établissements aux confins du Marais, "quartier endormi de vieux aristocrates ${ }^{37}$, tandis que Silvela s'installe à l'est du faubourg Saint-Antoine, quartier plus populaire où arrivent les migrants sans

34 Carola Reig Salvá, Vicente Salvá, un Valenciano de prestigio internacional, op. cit., p. 125. L'article d'Aline Vauchelle Haquet sur González Arnao ne fait aucune mention de cette activité : "Un afrancesado refugiado en Francia : Vincente González Arnao ", Trienio, Ilustración y liberalismo, n 9, 1987, p. 177-185.

35 AN, F/17/1407, déclarations prescrites par l'ordonnance du 16 juin 1828 de tous les chefs d'établissement de l'académie [de Paris] et de tous leurs répétiteurs, le 5 février 1829. Il s'agit des déclarations de non appartenance à une congrégation religieuse non autorisée à laquelle doivent se soumettre tous les professeurs exerçant dans des établissements d'enseignement.

36 Légerson, Agenda des pensions..., op. cit., 1836. À l'exception du Gymnase militaire du colonel Francisco de Amorós, Espagnol naturalisé français, dédié à la pratique de la gymnastique.

37 Bernard Marchand, Paris, histoire d'une ville, XIX ${ }^{e}-X X^{e}$ siècle, Paris, Seuil, 1993, p. 40. 
fortune $^{38}$. Ces établissements ne font pas partie du réseau institutionnel du ministère de l'Instruction publique : c'est "sous la protection des lois générales de la Police" que les établissements étrangers sont admis ${ }^{39}$, à l'exception du collège de Manuel Silvela qui obtient son rattachement à l'Université en 1828 .

Nous n'avons que peu de chiffres sur le taux de fréquentation de ces établissements, à l'exception de ceux cités plus bas pour le collège de Silvela. Nous ignorons également quelle part représentent les pensionnaires, de même que leur présence au sein des institutions françaises ${ }^{40}$. Ces établissements se distinguent surtout par leur recrutement original. Une partie des élèves est originaire des anciennes colonies espagnoles. Dans les jeunes républiques, le système éducatif n'est pas encore constitué. Les ressources manquent pour développer les écoles, les collèges, les universités et il y a peu de jeunes diplômés en mesure d'enseigner ${ }^{41}$. L'élite intellectuelle et économique se forme à Londres et surtout à Paris, capitales européennes d'où parviennent aussi la plupart des ouvrages édités en langue espagnole. La majorité des jeunes gens semble alors choisir la France catholique plutôt que sa voisine protestante ${ }^{42}$. La France encourage la venue des enfants de l'élite sud-américaine, hispanophone et brésilienne en leur accordant le transport gratuit ${ }^{43}$. En 1825, le ministre de la Marine et des Colonies met ainsi en avant les avantages qu'il y aurait pour l'avenir "à procurer aux familles les plus distinguées des nouveaux États de l'Amérique, des facilités pour faire élever leurs enfans en France ${ }^{44}$. En 1826, José Saëz, fils du secrétaire du ministre de la Guerre de la République du Chili, ainsi que Nicasio de Toro et Manuel Talavera, fils de riches négociants, viennent à Paris aux frais de la France :

"Les ministres ont reçu avec reconnoissance la grace que S.M. accorde aux jeunes Chiliens de les faire passer en France à ses frais, pour y aller puiser des connoissances qui manquent dans leur pays. Cinq ont déjà profité de ce bienfait,

38 Ibid.

39 AN, F/17/9044, dossier de Manuel Silvela. Manuel Silvela au grand-maitre de l'Université, ministre de l'Instruction publique, Paris, le 25 décembre 1827.

40 Aucune notice du ministère de l'Instruction publique sur l'un des établissements espagnols n'a été repérée.

41 Gregorio Weinberg, "Educación y sociedad", in Historia general de América latina, vol. 6 : La construcción de las naciones latinoamericanas, 1820-1870, Paris, Unesco Trotta, 2003, p. 573.

42 Thomas Calvo, L'Amérique ibérique de 1570 à 1910, Paris, Nathan, 1994, p. 297.

43 Delphine Diaz, "Des Brésiliens dans la France des années 1820. Contribution à une histoire des mobilités transatlantiques au XIX ${ }^{\mathrm{e}}$ siècle", Amnis, n 12 , 2013. En ligne : <http://amnis.revues. org/1919> (consulté le 29 mars 2014).

44 AN, F/17/9044, dossier de Manuel Silvela. Ministre de la Marine et des Colonies au ministre des Affaires ecclésiastiques et de l'Instruction publique, Paris, le 31 décembre 1827. 
et passent sur l'Arriège et le Lybio. Ils seront sans doute suivis par d'autres : les premières familles sentent la nécessité de l'instruction pour leurs enfans, et elles attachent d'autant plus de prix à la faveur qu'on leur accorde qu'elles sont certaines qu'ils seront élevés dans les principes de la vraie religion " ${ }^{45}$.

Les correspondants commerciaux s'assurent ensuite du bon déroulement du séjour. Les trois jeunes garçons "sont adressés à Paris, le premier à M. Larea, négociant rue Ste-Anne, et les deux autres à M. Gabriel Bardel, maison de MM. Sellières et Cie ${ }^{46}$. Parfois ce rôle est assuré par les chefs d'institution eux-mêmes $^{47}$.

\section{1 - La pension de l'abbé Prado puis de Vallejo, premier des établissements espagnols}

Le chanoine Gregorio Alonso de Prado, originaire de Valladolid, se réfugie en France en 1814 pour avoir soutenu la cause de Joseph Bonaparte. Il vit à Paris avec sa sœur, sa belle-sœur et sa nièce. En 1816, il ouvre une pension pour les jeunes Espagnols, tout en continuant à exercer une activité religieuse ${ }^{48}$. Il transmet les clés de son institution à José Mariano Vallejo en 1828, pour se consacrer alors à son ministère. Il est desservant à l'église Saint-Merri ${ }^{49}$ avant d'exercer comme diacre à Saint-Philippe-du-Roule en $1837^{50}$, date après laquelle nous perdons sa trace.

L'institution qu'il fonde est successivement située passage Petit SaintAntoine, rue de la Michodière, puis à partir de 1826, 6 rue de Beautreillis. Elle est d'abord destinée aux hispanophones, mais n'exclut pas les élèves d'autres origines. Parmi les professeurs figurent Joaquín Domingo Prior, José Mariano Vallejo $^{51}$, auteur de nombreux ouvrages éducatifs et successeur de Prado

45 AN, F/7/12060, Toro de Gusman, frères Saës, José, Bernardo et Alonzo Toro, Manuel Talavera. Ministre de la Marine et des Colonies au ministre de l'Intérieur, Paris, les 15 et 26 juin 1826. Rapport au ministre de l'Intérieur, Brest, le 23 juin 1826.

46 Ibid., ministre de la Marine et Colonies au ministre de l'Intérieur, Paris, le 15 juin 1826.

47 Juan Centeno, Francisco Agustín Silvela, Patorni, Précis pour M. Centeno, contre Salomon Béere et autres, Paris, Imprimerie Herhan, s. d.

48 AN, F/17/9044, dossier de Manuel Silvela. Manuel Silvela au grand-maitre de l'Université, Paris, le 25 décembre 1827.

49 AN, F/7/12076, Espagnols admis aux secours. Préfet de police au ministre de l'Intérieur, Paris, le 5 avril 1831. Ministre de l'Intérieur au préfet de police, Paris, le 19 mars 1831; AN, F/7/6642, "État nominatif des Espagnols arrivés à Paris (avril 1821)".

50 Archives historiques de l'archevêché de Paris, registre de conseil n ${ }^{\circ} 1$, conseil du 9 octobre 1837.

51 Plan de una casa de educación establecida en París, calle de Beautreillis $n^{\circ} 6$, destinada a los que 
en 1828, et le père Astete, auteur du Catecismo de la doctrina cristiana para el uso de la Casa de educacion establecida en Paris ${ }^{52}$. En 1827, ils sont rejoints par Domingo Fernández Ángulo qui crée vite son propre établissement, probablement lorsque Vallejo succède à Prado ${ }^{53}$. En 1828, l'équipe enseignante est composée d'Escriche, d'Ortega, de Baranda dont nous ignorons les spécialités, de Domingo Prior, professeur d'écriture, d’Hernáinz, professeur de chimie, et d'un Français, Frédéric Marchand ${ }^{54}$. Vallejo arrive fin 1824 à Paris. Alors présenté comme professeur de mathématiques par la police, il est accompagné de deux étudiants, Manuel de Navia, marquis de Santa Cruz de Marcenado, et son frère Benito María ${ }^{55}$. Sa situation politique paraît ambiguë puisqu'en 1825 il peut rentrer en Espagne pour raccompagner les deux étudiants avant de revenir à Paris où il monte un cabinet d'affaires avec Julián Rodríguez, un ancien professeur de sciences naturelles ${ }^{56}$ : "Ils se chargent [des affaires] que peuvent avoir à traiter dans la capitale ceux de leurs compatriotes qui y demeurent et ceux qui habitent les autres parties de la France et qui se trouvent même dans des pays étrangers ${ }^{57}$. À côté de l'activité de son cabinet, Vallejo donne des leçons. En 1826, après le décès de Rodríguez, il rejoint la maison d'éducation de Prado $^{58}$. Sa renommée s'étend jusqu'aux Français, puisqu'il donne aussi des leçons dans la maison du comte de Montmorency ${ }^{59}$. En 1828, il prend la direction de la pension de Prado. Son parcours avant l'exil explique vraisemblablement son investissement dans le champ de l'éducation :

hablan el idioma español o quieren aprenderlo y dirigida por D. Gregorio Alonso de Prado, eclesiástico español en unión con D. José Mariano Vallejo y D. Joaquín Domingo Prior, Paris, Imprimerie Farcy, 1826.

Catecismo de la doctrina cristiana para el uso de la Casa de educacion estallecida (sic) en Paris rue Beautreillis $n^{\circ} 6$, dirigida por D. Grégorio Alonso de Prado, Paris, Imprimerie Farcy, 1827.

53 Carola Reig Salvá, Vicente Salvá, un Valenciano de prestigio internacional, op. cit., p. 125.

54 AN, F/17/1407, déclarations prescrites par l'ordonnance du 16 juin 1829 de tous les chefs d'établissement de l'académie [de Paris] et de tous leurs répétiteurs, le 5 février 1829.

55 AN, F/7/ 12048, José Mariano Vallejo, Maria Benito de Navia, Manuel de Navia, marquis de Santa Cruz de Marcenado, Nicolas Barreto, comte de Casa-Barreto, comte de Selva Florida. Premier dossier. Note du directeur général de la police du royaume, d'après un état collectif de passeports transmis par le sous-préfet de Bayonne le 11 novembre 1824 et ministre de l'Intérieur au préfet de police, Paris, le 7 février 1825.

56 AN, F/7/3046/2, "Espagnols à Paris". s. d. [1808].

57 AN, F/7/12048, José Mariano Vallejo, Maria Benito de Navia, Manuel de Navia, marquis de Santa Cruz de Marcenado, Nicolas Barreto, comte de Casa-Barreto, comte de Selva Florida. Premier dossier. Cabinet du préfet de police au ministre de l'Intérieur, Paris, le 6 octobre 1825. Ibid., extrait d'un rapport du préfet de police au ministre de l'Intérieur, Paris, le 7 février 1826.

59 Ibid., cabinet du préfet de police au ministre de l'Intérieur, Paris, le 12 décembre 1826. Il n'a toutefois pas été possible d'identifier à quelle branche Montmorency la note du préfet fait allusion. 
"Cet étranger était, en 1814, professeur de sciences exactes au séminaire des nobles à Madrid. Il se fit remarquer en 1820 par son exaltation révolutionnaire et obtint l'emploi de directeur de l'instruction publique, titre qu'il a pris encore dans une circonstance récente, quoiqu'il ait été privé à la restauration de S.M.C. de toute charge publique $"^{60}$.

Le plus ancien des établissements espagnols semble être le premier à disparaître. En 1829, Vallejo entreprend une série de voyages en Europe avant de rentrer définitivement en Espagne en 1832, où il se consacre aux sciences et participe à l'organisation du système d'études espagnol ${ }^{61}$. Malgré une direction assez courte, José Mariano Vallejo laisse sa marque dans l'éducation des jeunes Espagnols de Paris en publiant plusieurs manuels de lecture et de mathématiques, tels que les Ideas primarias que deben darse a los niños en las escuelas acerca de los números et le Compendio de matemáticas puras y mixtas ${ }^{62}$.

\section{2 - Le collège de Silvela, une éphémère réussite financière et politique}

En 1813, l'afrancesado Manuel Silvela García de Aragón, ancien professeur de droit à l'université de Valladolid ${ }^{63}$, se réfugie avec sa famille près de Bordeaux. En 1816, il y fonde un collège pour les Espagnols et les Américains, fréquenté par une vingtaine d'élèves ${ }^{64}$, couramment appelé "collège" ("colegio" en espagnol), ou "école hispano-lusitanienne "65. D’autres réfugiés y enseignent, comme le dramaturge Leandro Fernández de Moratín ou Pablo de Mendíbil qui publie, en collaboration avec Silvela, un manuel scolaire, Biblioteca selecta de literatura española ${ }^{66}$. Lors de la révolution libérale de 1820, Mendíbil rentre en Espagne,

60 Ibid., ministre de l'Intérieur au ministre des Affaires étrangères, Paris, le 7 janvier 1826.

61 Alberto Gil Novales (dir.), Diccionario biográfico del Trienio liberal, Madrid, El Museo universal, 1991, notice "Vallejo, José Mariano», p.670-671.

62 José Mariano Vallejo, Ideas primarias que deben darse a los niños en las escuelas acerca de los números al mismo tiempo que se estan ejercitando en la clave analitica de la lectura, Paris/Mexico, Bossange, 1826; José Mariano Vallejo, Compendio de matemáticas puras y mixtas, Paris, Bossange, 1826.

63 En ligne : <https://www.museodelprado.es/coleccion/galeria-on-line/galeria-on-line/obra/ manuel-silvela/> (consulté le 18 octobre 2014).

64 AN, F/7/12043, d. Manuel Garcia Silvela de Aragon. M. Silvela au ministre de l'Intérieur, Paris, le 27 septembre 1827.

65 Par exemple : Revue encyclopédique ou analyse raisonnée des productions les plus remarquables dans les sciences, les arts industriels, la littérature et les beaux-arts, Paris, Bureau central de la revue encyclopédique, chez Sédillot, libraire, t.44, 1829, p.493-495 [Recension de Mac Culloch's principles of political economy abridged for scholar usage at Dr. Silvela's hispano-lusitanian institution par John Ramsay Mac Culloch].

66 Pablo de Mendíbil, Manuel Silvela, Biblioteca selecta de literatura española, ó Modelos de elocuencia 
mais doit de nouveau s'exiler en 1823. Il choisit Londres où il enseigne le français et l'espagnol jusqu'à sa mort en $1832^{67}$. Fernández de Moratín et Manuel Silvela choisissent de rester en France en 1820. En juin 1827, sur une proposition de l'abbé Prado qui cherche probablement déjà un successeur, Silvela arrive à Paris. Pour des raisons inconnues, la fusion des deux institutions n'est pas réalisée. C'est dans ce contexte que Silvela organise alors le transfert de son collège à Paris, demandé "par plusieurs pères de famille" ${ }^{68}$. Silvela trouve un local au numéro 33 de la rue de Montreuil en septembre 1827 :

"Le premier batiment, se compose d'un vaste rez de chaussée, d'un premier et d'un second étage avec grenier au dessus suivant l'etat des lieux fait double entre les parties.

Le deuxième batiment qui est à gauche se compose également d'un rez de chaussée, d'un premier et second étage.

À la suite de la cour et séparé par une grande grille en fer se trouve un jardin d'agrément "69.

Outre les salles de cours et les dortoirs, il y a une bibliothèque et un cabinet de physique et de minéralogie. Dans l'attente de la construction d'une chapelle, les élèves vont à la messe à l'église paroissiale de Sainte Marguerite ${ }^{70}$.

Les élèves de Bordeaux, encadrés par Fernández de Moratín, arrivent à Paris pour la rentrée scolaire, en octobre $1827^{71}$. Ils bénéficient de cours diversifiés, comme le montre le tableau ci-après ${ }^{72}$ :

y poesía tomados de los escritores mas célebres desde el siglo XIV hasta nuestros días, Bordeaux, Imprimerie Lawalle, 1819, 4 vol.

67 Manuel Valera Candel, "Actividad científica realizada por los liberales españoles exiliado en el Reino Unido, 1823-1833", Asclepio. Revista de Historia de la Medicina y de la Ciencia, vol. 59, 2007, n 1 , p. 131.

68 AN, F/7/12043, d. Manuel Garcia Silvela de Aragon. Manuel Silvela au ministre de l'Intérieur, Paris, le 27 septembre 1827.

69 AN, F/17/9044, dossier de Manuel Silvela. Copie du bail signé entre Silvela et M. Leroux, Paris, le 12 septembre 1827.

70 Ibid., rapport de M. L’Étendard, inspecteur, Paris, février 1828.

71 Ibid., ministre de l'Intérieur au ministre des Affaires ecclésiastiques et de l'Instruction publique, Paris, le 5 décembre 1827.

72 AN, F/17/1407, déclarations prescrites par l'ordonnance du 16 juin 1828 de tous les chefs d'établissement de l'académie [de Paris] et de tous leurs répétiteurs, 5 février 1829. 


\begin{tabular}{l|l}
\hline Cours & Enseignant \\
\hline Philosophie et histoire & Manuel Silvela \\
\hline Latin & Francisco Agustín Silvela (fils de Manuel Silvela) \\
\hline Français & Eugène Menoz \\
\hline Écriture & Jean-Jacques-Achille Lachave $^{73}$ \\
\hline Mathématiques & Jules Planche $^{74}$ \\
\hline Sciences naturelles & Andrés Alcón \\
\hline Géographie & Figueira (beau-fils de Silvela) \\
\hline $\begin{array}{l}\text { Économie politique, droit public } \\
\text { et législation sociale }\end{array}$ & Silvestre Pinheiro Ferreira \\
\hline Dessin & Raymond Bonheur \\
\hline Comptabilité & Vertamy \\
\hline
\end{tabular}

Les cours du collège de Silvela et leurs enseignants en 1829

Une lettre de Manuel Silvela, adressée au ministre de l'Instruction publique en 1827 pour demander la reconnaissance officielle de l'établissement, permet de connaître l'esprit de cet enseignement :

"Dans un établissement de cette nature, tout ce qui n'est pas la langue française doit être espagnol, c'est le seul lien qui nous unisse. Pour la partie religieuse, prières, catéchisme, tout est différent, et c'est dans le langage de la religion que l'homme ne doit jamais être dépaysé. Dans ses relations avec la Divinité le langage le plus connu, le mieux senti suffit à peine pour exprimer la sublimité de tels sentiments $"^{75}$.

Par conviction mais probablement autant par obligation, Silvela insiste sur la place de la religion, dispensée en latin, comme dans les établissements français. De par sa formation universitaire, il est certainement sensible à cette question : en 1806, il a obtenu à Valladolid son doctorat en jurisprudence, en philosophie et en théologie. Il est également licencié en droit canonique ${ }^{76}$. Par ailleurs, l'enseignement de la religion catholique est, nous l'avons vu, un argument de

73 Inventeur d'une tablette d'écriture destinée à l'enseignement primaire (Bulletin du ministère de l'Agriculture et du Commerce, partie officielle, Paris, Librairie et imprimerie administratives, 1842, p. 208).

74 Celui-ci enseigne également au collège royal Charlemagne. Agrégé ès sciences à Paris en 1825 (<http://rhe.ish-lyon.cnrs.fr/?q=agregsecondaire_laureats>, consulté le 17 décembre 2014).

75 AN, F/17/9044, dossier de Manuel Silvela. Manuel Silvela au grand-maître de l'Université, ministre de l'Instruction publique, Paris, le 25 décembre 1827.

76 Alberto Gil Novales (dir.), Diccionario biográfico del Trienio liberal, op. cit., notice "Silvela García de Aragón, Manuel», p. 625. 
poids pour attirer la jeunesse sud-américaine à Paris. Mais surtout, le destinataire de la lettre de Silvela est $\mathrm{M}^{\text {gr }}$ Frayssinous, grand-maitre de l'Université, ministre des Affaires ecclésiastiques et de l'Instruction publique depuis 1824 et qui favorise la transformation en collèges officiels des établissements privés qui méritent "la confiance des familles par leur direction religieuse et la force de leurs études „"77. Par un arrêté du 18 mars 1828, Silvela obtient cette autorisation, d'abord "à la condition expresse de ne recevoir que des Espagnols dans son établissement ${ }^{78}$. Le reste des cours dispensés dans le collège de Silvela semble établi en parallèle de ceux des établissements français :

"Dans la partie scientifique, c'est en espagnol qu'on doit tout enseigner [...]. Dans un système d'enseignement fait pour des Français, on fait parcourir à l'élève pas à pas, tous ses départements, tandis que pour des Espagnols ce sont nos provinces, nos colonies, l'Amérique du nord et du sud, qu'il faut lui faire parcourir en détail. Dans l'histoire, ce n'est pas l'histoire de France, mais la nôtre qui doit occuper pour nous la première place. Dans les belles lettres, à commencer par la langue latine, celle de la Castille doit être exclusive pour des Espagnols, le terme de comparaison pour les rudiments, doit être la langue castillane, notre manière de lire est tellement différente, qu'on prendrait presque pour deux morceaux dans deux langues diverses, un même passage de Virgile lu par un Français puis par un Espagnol; c'est enfin en espagnol que l'on doit faire les premières versions, les traductions et les compositions. En rhétorique nous admirons le siècle de Louis XIV, mais c'est en espagnol qu'on doit faire les explications, et c'est dans nos classiques, qu'il faut puiser les exemples. C'est là que nous devons trouver nos modèles "79.

Pour Silvela, les mathématiques sont, après la religion, l'enseignement le plus important : il favorise la victoire de la raison sur la passion. Né en 1781, ancien afrancesado, il est davantage attiré par les sirènes du rationalisme d'un Nicolas de Condorcet que par les premiers frémissements romantiques de Germaine de Staël. Mais, malgré son attachement aux idées révolutionnaires françaises, par son établissement et par l'envergure qu'il lui donne, il se pose également en défenseur de la langue, de l'histoire et de la culture espagnoles, encore méconnues en France.

En janvier 1828, l'établissement accueille vingt-sept élèves ${ }^{80}$, en 1830, plus

77 Francis Démier, La France de la Restauration (1814-1830). L'impossible retour du passé, Paris, Gallimard, 2012, p.741-742.

78 AN, F/17/9044, dossier de Manuel Silvela. Rapport. s. d.

79 Ibid., Manuel Silvela au grand-maitre de l'Université, ministre de l'Instruction publique, Paris, le 25 décembre 1827.

80 AN, F/7/12043, d. Manuel Garcia Silvela de Aragon. Préfet de police au ministre de l'Intérieur, Paris, le 11 janvier 1828 . 
de $180^{81}$. En novembre 1830, l'autorisation accordée à Silvela par le ministère de l'Instruction publique d'"admettre des élèves sans distinction du pays auquel ces élèves appartiennent „ 82 sanctionne sa réussite. Son collège initialement réservé aux Espagnols semble connaître un grand succès auprès de la jeunesse hispano-américaine, comme en témoigne une lettre de Vicente Pérez Rosales, représentant du Chili, au ministre de la Marine et des Colonies :

"J'ai d'autant plus d'intérêt à ce que M. Silvela soit autorisé à fourmer (sic) son institution, que je lui ai déjà confié douze jeunes Chiliens à l'éducation desquels je suis chargé de veiller, et que, sur ma recommandation, le Ministre du Pérou à Londres se dispose à y envoyer douze autres jeunes gens dont l'éducation doit être faite aux frais du gouvernement péruvien. En outre, la maison de M. Silvela présentant la réunion des études les plus utiles dans tous les genres, je ne doute pas que mes compatriotes et les familles des contrées voisines du Chili, sûres de trouver ici un établissement précieux pour leurs enfants, ne s'empressent de les y envoyer faire leur éducation, en adoptant pour cela exclusivement la France ${ }^{83}$.

Les étudiants portugais sont aussi les bienvenus comme l'indique le nom de l'institution et la présence de deux enseignants portugais. À l'instar de Vallejo, Manuel Silvela est un pédagogue et publie plusieurs manuels scolaires, destinés à compléter l'éducation dispensée dans son établissement, comme le Compendio de la historia romana hasta los tiempos de Augusto ${ }^{84}$.

Le collège de Silvela se distingue des autres établissements espagnols parce qu'il est officiellement reconnu et financé par l'Université. Les élèves espagnols et américains sont autorisés à fréquenter les collèges royaux et à passer les examens pour l'obtention des diplômes français ${ }^{85}$. Cette autorisation est sans doute l'une des clés du succès. Le collège de Silvela est le plus prestigieux des établissements qui accueillent des élèves hispanophones. Mais en 1832, une ordonnance du roi d'Espagne Ferdinand VII, en interdit l'accès aux Espagnols :

81 Raül Veiga, "Hispano-americanas en Francia: la Restauración y América independiente. ¿Realismo o dogmatismo?", in Voyages et séjours d'Espagnols et d'Hispano-Américains en France, Tours, Publications de l'université de Tours, 1982, p. 14.

82 AN, F/17/9044, dossier de Manuel Silvela. Extrait du registre des délibérations du Conseil royal de l'Instruction publique, procès-verbal de la séance du 6 novembre 1830.

83 Ibid., J.X. Rosales au ministre de la Marine et des Colonies, Paris, le 27 décembre 1827. Copie.

84 Manuel Silvela, Compendio de la historia romana hasta los tiempos de Augusto, Paris, Imprimerie Gaultier-Laguionie, 1830.

85 AN, F/17/9044, dossier de Manuel Silvela. Manuel Silvela au ministre de l'Instruction publique, Paris, le 18 octobre 1830 . 
"Ayant vu récemment dans mon conseil des ministres une procédure relative à l'éducation que reçoivent à Paris, dans un collège tenu par un sieur Silvela, différents jeunes Espagnols et Américains, avec l'avis de mon conseil, j'ai ordonné que l'on renouvelât les ordres expédiés antérieurement afin que l'on ne permette point aux jeunes gens de la péninsule et des Amériques fidèles d'aller faire leur éducation dans les collèges de France, où en outre de la société des jeunes français de leur âge et de la contagion si facile dans les premières années inexpérimentées de la vie des principes de politique repoussés par mes vassaux d'Espagne, ils ont pour condisciples beaucoup de jeunes gens de provinces d'Amérique insurgées " 86 .

L'établissement n'est pas pour autant une pépinière de libéraux. Le sursaut de Ferdinand VII est tardif. Silvela semble d'abord avoir bénéficié d'une certaine bienveillance de la part des diplomates espagnols. Sous la Restauration, la direction religieuse des élèves est en effet assurée par l'aumônier de l'ambassadeur d'Espagne ${ }^{87}$. La visite du général José de San Martín, héros de l'indépendance argentine, au collège de Silvela en 1829 a probablement augmenté la notoriété de l'établissement en Amérique latine et cristallisé l'opposition des Espagnols : "La présence de San Martín dans le collège a causé aux Chiliens et aux Argentins la plus vive joie, aux Péruviens, de la taciturnité et aux Espagnols du mécontentement " ${ }^{88}$. Silvela sait aussi s'adapter aux circonstances. Sous la Restauration, malgré son passé d'"afrancesado", il apparaît comme une figure rassurante pour les autorités : "Il a le but très louable de tempérer les têtes un peu exaltées de ces Américains, appartenant la plûpart aux anciennes colonies espagnoles, qui se sont aujourd'hui déclarées indépendantes de la mère-patrie " 89 . Sous la monarchie de Juillet, le ton des missives qu'il adresse aux nouvelles autorités françaises change. Il n'hésite pas à remettre en cause le pouvoir en place en Espagne : "Cette jeunesse américaine surtout avide de science cherche une éducation espagnole, mais libre des entraves que le gouvernement de la péninsule impose au développement des lumières, et cette éducation elle la trouve dans la capitale de la France, au centre de la civilisation et du goût, elle

86 Archives du ministère des Affaires étrangères. Mémoire et documents Espagne, 310, nº 41 : traduction d'une ordonnance de Ferdinand VII datée de Madrid, le 17 février 1832.

87 AN, F/17/9044, dossier de Manuel Silvela. Rapport de M. L'Étendard, inspecteur, Paris, février 1828.

88 Vicente Pérez Rosales, Recuerdos del pasado (1814-1860), édition en ligne par Patrick Barros, <http://www.librosmaravillosos.com/recuerdosdelpasado/capitulo05.html> (consulté le 26 avril 2014). Traduction.

AN, F/17/9044, dossier de Manuel Silvela. Rapport de M. L'Étendard, inspecteur, Paris, février 1828. 
la trouve mêlée à l'éducation française $"^{90}$. Silvela ne cache plus son admiration pour la philosophie des Lumières.

Le fait que seul le collège de Silvela soit cité par l'ordonnance de Ferdinand VII de 1832 pourrait laisser penser que les deux autres établissements espagnols, celui de José Mariano Vallejo et celui de Domingo Fernández Ángulo, n'existent plus, même s'ils n'ont jamais eu la même importance ni bénéficié de la même publicité. Manuel Silvela décède le 9 mai 1832. Son fils Francisco Agustín Silvela lui succède au moins jusqu'en 1833 comme le prouve le Précis pour M. Centeno contre Salomon Béere et autres, publié la même année. Il s'agit d'une sorte de mémoire en défense de Silvela fils et de l'avocat Patorni en faveur d'un jeune pensionnaire péruvien du collège, Juan Centeno, abusé par des commerçants. Mais, peu de temps après le décès de Ferdinand VII, en septembre 1833, Francisco Agustín rentre en Espagne où il entreprend une carrière d'homme politique et de juriste ${ }^{91}$. Guant à l'établissement de la rue de Montreuil, son usage scolaire demeure, mais il ne paraît plus s'adresser spécifiquement à la jeunesse hispanophone. En 1835, c'est un dénommé Mailhat qui est maitre de pension à cette adresse ${ }^{92}$.

\section{3 - Le collège de Domingo Fernández Ángulo, entre investissement financier et projet pédagogique}

Domingo Fernández Ángulo arrive à Paris en novembre 1824, bientôt rejoint par sa femme et ses quatre enfants. Négociant d'Oviedo, âgé d'une quarantaine d'années ${ }^{93}$, il était auparavant correspondant en Espagne de Belrichard, libraire de Bayonne, spécialisé dans l'édition d'ouvrages en langue espagnole "séditieux" qu'il faisait entrer en contrebande. Fernández Ángulo, qui appartient selon la police aux "hauts grades de la maçonnerie", a participé à la rédaction d'un journal, l'Expectador, pendant le "Trienio liberal". Homme instruit, parlant très bien le français, ses premières ressources à Paris proviennent de la traduction d'ouvrages sur la Révolution française, à destination du Mexique, pour le compte de Joaquín María Ferrer y Cafranga ${ }^{94}$, ancien député de Guipúzcoa ${ }^{95}$. Cette activité n’appa-

90 Ibid., Manuel Silvela au ministre de l'Instruction publique, Paris, le 18 octobre 1830.

91 En ligne :<http://www.senado.es/web/conocersenado/senadohistoria/senado18341923/senadores/ fichasenador/index.html?id1=2797 > (consulté le 10 mai 2014).

92 Almanach royal et national pour l'an 1835, Paris, Guyot et Scribe, 1835, p. 873.

93 AN, F/7/12048, Domingo Fernandez de Angulo. Note du ministre de l'Intérieur, Paris, le 27 novembre 1824 .

94 Ibid., cabinet du préfet de police au ministre de l'Intérieur, Paris, le 6 janvier 1825.

95 Ibid., Domingo Fernandez de Angulo. Préfet de police au ministre de l'Intérieur, Paris, le 18 mai 1827. 
raît toutefois pas très rentable ${ }^{96}$. À partir de 1827, il devient professeur dans la maison d'éducation de Prado ${ }^{97}$. Nous ignorons la ou les disciplines qu'il enseigne.

En 1828, il crée son propre établissement, le "collège étranger", peut-être avec le soutien des Bertrán de Lis, famille de négociants et financiers ${ }^{98}$, rue SaintGilles, non loin de l'établissement de Prado ${ }^{99}$. À l'exception de Fernández Ángulo et d'un ecclésiastique nommé Juan González Cabo-Reluz, docteur en théologie, professeur d'histoire et de "sciences philosophiques", l'ensemble du corps enseignant est français et dispose de bonnes références : Pierre-Henri Suzanne, professeur de mathématiques ${ }^{100}$, exerce déjà au collège royal Charlemagne et est l'auteur en 1830 de Principes élémentaires d'algèbre, ouvrage spécialement dédié au collège étranger dirigé à Paris par D.F. Angulo101, Frédéric Marchand, professeur de français, enseigne aussi à la pension de Vallejo, Jean-Jacques Colin ${ }^{102}$, professeur de chimie et de physique, enseigne à l'école militaire de Saint-Cyr, enfin, Jean-Louis Fouqueur, professeur de dessin et d'écriture, travaille aussi à son compte ${ }^{103}$. L'établissement semble d'abord destiné aux enfants des Espagnols émigrés politiques puisque une réclame est publiée lors de son ouverture dans El emigrado observador, un périodique destiné aux Espagnols réfugiés en Grande-Bretagne et en France, qui y consacre également un article. Il vise également les enfants des "nouvelles nations américaines" hispanophones voire les jeunes Anglais qui veulent s'instruire dans une autre langue ${ }^{104}$.

Sur un ton rousseauiste, le "collège étranger" y est décrit comme un vaste et magnifique édifice avec un jardin dont les dimensions assurent le confort et la joie nécessaires à la santé des élèves. La nourriture y est saine et abondante. Quant aux matières enseignées, une place d'honneur est accordée à la religion,

96 Ibid., Domingo Fernandez de Angulo. Préfet de police au ministre de l'Intérieur, Paris, le 5 février 1825.

97 Ibid., préfet de police au ministre de l'Intérieur, Paris, le 16 février 1828.

98 Carola Reig Salvá, Vicente Salvá, un Valenciano de prestigio internacional, op. cit., p. 125.

99 AN, F/17/1407, déclarations prescrites par l'ordonnance du 16 juin 1828 de tous les chefs d'établissement de l'académie [de Paris] et de tous leurs répétiteurs, le 5 février 1829.

100 Base QUIDAM des Archives nationales. Consultable en salles de lecture.

101 Ouvrage cité dans la Bibliographie de la France ou journal général de l'imprimerie et de la librairie, $\mathrm{n}^{\circ} 7,13$ février 1830, p. 106.

102 Auteur d'un Cours de chimie à l'usage de MM. les élèves de l'École militaire de Saint-Cyr, Paris, Thomine, 1827.

103 AN, F/17/1407, déclarations prescrites par l'ordonnance du 16 juin 1828 de tous les chefs d'établissement de l'académie [de Paris] et de tous leurs répétiteurs, le 5 février 1829, et Guyot de Fère, Annuaire des artistes français, Paris, Guyot de Fère, Audot, Gosselin, 1832, p. 75.

104 El Emigrado observador, n5, novembre 1828, p. 186-187. 
même si elle n'est considérée que comme une partie de l'éducation morale. Les matières proposées sont nombreuses et marquées par les idées et la sociologie des exilés espagnols : arithmétique, grammaire espagnole, français, anglais, italien et latin, littérature, géographie, chronologie et histoire ancienne et moderne, philosophie "rationnelle" et morale, économie politique, droit naturel, droit des gens et droit public, mathématiques au sens large, physique, chimie, botanique, minéralogie, comptabilité, dessin, danse, escrime et musique. Les petits Espagnols doivent acquérir un bagage qui leur permettra non seulement de poursuivre le combat de leurs pères mais également de survivre en exil, grâce à l'apprentissage des langues étrangères et à l'acquisition de compétences pratiques. L'importance apportée au maintien de la santé physique fait écho à l'œuvre de l'Espagnol Francisco Amorós, introducteur de la gymnastique et de l'éducation physique en France, pratiques fondées sur les écrits de Johann Heinrich Pestalozzi ${ }^{105}$. Les frais de scolarité s'élèvent à 1500 francs annuels, pension non incluse, auquel il faut ajouter 200 francs de frais d'inscription ainsi qu'un certain nombre d'activités optionnelles : dessin, musique, danse, escrime, équitation, gymnastique et "taquigraphie" ( $(\mathrm{cic})^{106}$. Il est aussi possible d'y loger (pour un tarif allant de 1500 à 2000 francs annuels), sans suivre les enseignements. Pour donner un ordre de grandeur, le gouvernement français attribue à la même époque un subside de 1200 francs annuels au général Francisco Valdés, une des figures de premier plan de l'exil espagnol ${ }^{107}$. Les tarifs semblent élevés comparés à ceux pratiqués par d'autres institutions, davantage établies, de la capitale. L'école spéciale de commerce et d'industrie, installée dans l'hôtel de Sully, rue Saint-Antoine au cœur du Marais, et qui emploie plusieurs personnalités prestigieuses telles que Jacques Laffitte ou Charles Dupin, propose au début des années 1840 un tarif annuel de 1400 francs incluant la pension et la plupart des enseignements (les cours de dessin "linéaire" et de chimie coûtent 5 francs supplémentaires chacun) ${ }^{108}$. En dépit de ces ambitions, l'établissement de Fernández Ángulo disparaît vite. Nous n’avons

105 Marc Le Cœur, "Couvert, découvert, redécouvert... L'invention du gymnase scolaire en France (1818-1872)", Histoire de l'éducation, nº 102, mai 2004, p. 109-135.

106 La tachygraphie est un "système d'écriture rapide utilisant un alphabet conventionnel ou un système de signes tel la sténographie", <http://www.cnrtl.fr/definition/tachygraphie > (consulté le 11 octobre 2014).

107 AN, F/7/12101, Francisco Valdés. Ministre de la Guerre au ministre de l'Intérieur, Paris, le 8 avril 1831.

108 Édouard Charton (dir.), Guide pour le choix d'un état ou dictionnaire des professions, Paris, Librairie veuve Lenormant, 1842, p. 233 et 235. 
aucune information sur son fonctionnement. Il ne figure pas dans l'Almanach du commerce de 1833. Profitant vraisemblablement d'une amnistie, Fernández Ángulo rentre en Espagne au début de la décennie $1830^{109}$. Contrairement à Gonzálo Cabo-Reluz, le professeur d'histoire du collège, ou à Vallejo, son retour en Espagne ne suscite pas d'investissement dans le monde scolaire ou universitaire ou dans le champ de la réflexion pédagogique. En 1840, il publie un ouvrage sur la nécessité de réformer le système fiscal espagnol ${ }^{110}$.

Il est en revanche certain que l'évolution politique espagnole permet le retour au pays d'une partie des fondateurs et des professeurs de ces établissements. Toutefois, leur disparition est brutale. La brièveté de l'existence de ces établissements et leur simultanéité semblent résulter de la combinaison de la présence des réfugiés espagnols à Paris et d'une forte demande sud-américaine. Les jeunes Hispano-américains viennent à Paris pour bénéficier de la qualité de l'instruction française. Les établissements espagnols ont donc été pour eux des structures transitoires : comme lieu d'hébergement et d'accueil, sur le mode des collèges par nation d'Ancien Régime, et comme écoles préparatoires aux classes françaises. Ils auraient pu évoluer vers des fondations comme il en existe alors pour les réfugiés polonais, à l'instar de l'école préparatoire polonaise de Stanislas Gajewski, financée par le prince Czartoryski et subventionnée par le ministère de l'Intérieur français ${ }^{111}$. Mais les établissements de ce genre s'adressent plutôt aux étudiants nécessiteux, ce qui ne correspond pas à la clientèle des institutions espagnoles créées sous la Restauration. Par ailleurs, les conflits propres à l'Amérique du Sud ainsi que la montée d'une certaine méfiance quant à l'envoi des jeunes gens en Europe - au point qu'on prescrit aux parents de "retarder le voyage initiatique jusqu'à la maturité ${ }^{112}$ - ont probablement favorisé des formes d'accueil et d'accompagnement plus individualisées, sur le mode du tutorat, rôle notamment joué par les correspondants commerciaux ou consulaires en Europe.

109 Alberto Gil Novales (dir.), Diccionario biográfico del Trienio liberal, op. cit., notice «Fernández de Angulo, Domingo", p. 225.

110 Proyecto de un nuevo sistema de contribuciones en España : adecuado a las penosas circunstancias en que nos hallamos, capaz de producir al Estado todo lo necesario, al mismo tiempo que liberte a la nación de todo impuesto oneroso, y propio para fomentar rápidamente la agricultura, el comercio y las artes, Madrid, Miguel de Burgos, 1840.

111 AN, F/17/9045, ministère de l'Instruction publique et des Cultes. Académie départementale de la Seine, notice de l'École préparatoire polonaise, Paris, février 1854.

112 Isabelle Tauzin-Castellanos, "L’Amérique latine et la passion du français au XIX siècle", Actes du Ve congrès de la Société des études romantiques et dix-neuviémistes, 24-26 janvier 2012, <http:// etudes-romantiques.ish-lyon.cnrs.fr/wa_files/Langues-Tauzin.pdf> (consulté le 10 mai 2014). 


\section{III - Émigration politique et pédagogie}

La brièveté de l'existence de ces établissements semble correspondre au calendrier de la vie politique espagnole, de même que le dynamisme de l'édition en langue castillane, en particulier des manuels de langue. Le secteur de l'éducation et la réflexion pédagogique sont davantage investis par les Espagnols réfugiés plutôt que par les migrants de la péninsule établis à Paris dans d'autres circonstances. Les réfugiés ne constituent pas la majorité de la population espagnole mais, dès leur arrivée, ils doivent trouver rapidement un moyen de subsistance ${ }^{113}$. La sociologie de l'émigration espagnole dans la capitale ne reflète nullement celle des réfugiés en France dans leur ensemble, car les professions intellectuelles y sont surreprésentées. Les réfugiés, chassés pour leurs idées, s'attachent à expliquer leur combat à leur propre jeunesse et s'interrogent sur la meilleure façon de transmettre ces valeurs afin de ne pas connaître d'autres échecs. Certains estiment que la pédagogie peut constituer une véritable arme de combat à retardement. Défenseurs du libéralisme, ils souhaitent offrir la possibilité aux enfants espagnols d'une formation complète et équilibrée, tant sur le plan des connaissances que des valeurs morales ou de l'entraînement physique, pour en faire des hommes meilleurs. En 1826, Cecilio de Corpas, absolutiste infiltré dans le milieu libéral parisien, traduit en espagnol La morale en action, ou meilleure sélection de faits mémorables et d'anecdotes instructives, propres à rendre aimable la sagesse, et à former le cœur de la jeunesse avec l'exemple de toutes les vertus, illustrées avec des passages historiques, de Laurent-Pierre Bérenger ${ }^{114}$. Les réflexions préliminaires donnent le ton : "Un enfant, éduqué avec les précautions que nous désirons, doit chercher, très vite, par une noble émulation, le moyen de ressembler aux modèles que nous lui présentons ${ }^{115}$. Les exemples devant montrer le chemin de vertus telles que la tempérance, la clémence ou la générosité ne sont plus

113 Les recensements de population ne prennent en compte la population étrangère qu'à compter de 1851, date à laquelle 1351 Espagnols sont recensés à Paris : Jacques Grandjonc, "Les étrangers à Paris sous la Monarchie de Juillet et la Seconde République", Population, n² 29, 1974, p. 63.

114 ANF F/7/12032, d. Francisco Xavier de Burgos, Doña de Olava, épouse du général Escudero. Extrait du rapport du préfet de police du 11 mars 1826.

115 La moral en accion, ó Lo mas selecto de hechos memorables y anécdotas instructivas, propias á hacer amable la sabiduría, y á formar el corazon de la juventud con el ejemplo de todas las virtudes, instruyéndolas con pasages históricos. Obra útil para los niños de las Escuelas y Casas de educacion de uno y otro sexo. Traducida del frances por Cecilio de Corpas, Paris, Masson, 1823, p. 11. Traduction. 
tirés du corpus biblique mais de l'histoire, ce qui doit susciter l'émulation des jeunes Espagnols. D’autres réfugiés, comme Vallejo et Silvela, ont de véritables préoccupations pédagogiques, comme en témoignent leurs travaux, cités plus haut. En 1829, Silvela s'explique, dans un texte imprimé, directement auprès de ses jeunes étudiants: "J'ai voulu recueillir dans une certaine mesure les maximes, les préceptes que je souhaiterais graver dans vos cœurs, pour que l'artifice de la métrique qui facilite la mémorisation, contribue à rendre éternelles les impressions de la vertu "116. Les pédagogues en exil profitent aussi de la possibilité de publier facilement à Paris le fruit de leur réflexion. Les réfugiés bénéficient donc, de manière générale, d'un contexte favorable pour développer leur réflexion sur les vertus comparées de différentes pédagogies et systèmes éducatifs : en expérimentant "grandeur nature" leurs idées, en se confrontant à d'autres réalités. Sebastián de Miñano y Bedayo estime d'ailleurs que l'émigration en soi est une forme d'apprentissage :

"Il n'y a pas d'émigré, aussi rude et peu appliqué qu'il soit, qui n'a pas fait, de gré ou de force, une foule d'observations et de comparaisons qu'à son retour il applique ou communique avec une certaine vanité à ses amis et ses compatriotes. L'un observe le progrès ou le retard des arts mécaniques; l'autre les coutumes domestiques et familiales; celui-ci se met à traduire, bien ou mal, les livres dont il croit qu'ils pourront être utiles ou du moins, se vendre dans son pays; certains étudient les méthodes les plus profitables à l'une ou l'autre branche de l'industrie ou de l'agriculture; nombre d'entre eux se consacrent à l'enseignement de leur propre langue et l'étudient en même temps; d'autres suivent les enseignements organisés dans les villes où la chance ou leur situation particulière leur a permis de résider ${ }^{117}$.

Sur un autre plan, sous la monarchie de Juillet, une partie de l'élite libérale réfugiée fréquente les mêmes cercles que les nouveaux hommes de pouvoir en France : l'homme de lettres et ancien diplomate Francisco Martínez de la Rosa, futur ministre, est un familier de François Guizot, instigateur de la loi du 28 juin 1833 sur l'instruction primaire en France ${ }^{118}$, dont le but est de mettre en place un "gouvernement des esprits" et, sans remettre en question l'ordre social existant, d'assurer une instruction minimale à l'ensemble de la

116 Manuel Silvela, En mi cumpleaños de mil ochocientos veinte y nueve, Paris, Imprimerie GaultierLaguionie, 1829, p.3-4. Traduction.

117 Sebastián de Miñano y Bedoya, "Emigraciones, emigrados », Revista de la Civilización európea, t. 6, juin 1843, p. 179-180. Traduction.

118 Jean Sarrailh, Un homme d'État espagnol : Martínez de la Rosa (1787-1862), Paris, E. de Boccard, 1930, p. 277. 
population : "développer l'intelligence, propager les lumières, c'est assurer l'empire et la durée de la monarchie constitutionnelle " ${ }^{119}$. Cette préoccupation politique rejoint celle des réfugiés espagnols : d'une part, enraciner les idées libérales au sein même de la population émigrée, en particulier auprès de la jeunesse; d'autre part, diffuser les lumières en Espagne afin que la population adhère aux principes généraux de la monarchie constitutionnelle.

L'expérience des établissements espagnols et les manuels imprimés témoignent de l'importance de la démarche pédagogique des réfugiés. Pour autant, il n'est pas aisé d'évaluer leur influence sur l'évolution de l'enseignement en Espagne à leur retour d'exil. Lorsque les libéraux arrivent au pouvoir en Espagne, ils investissent progressivement le champ de l'éducation. Entre 1835 et 1849 , cinquante-neuf lycées sont créés ${ }^{120}$. Les années 1830 et 1840 voient la promulgation de différents plans d'éducation. En 1836, le plan général d'instruction publique du duc de Rivas, non appliqué, rappelle la loi française de 1833 : il accorde la gratuité de l'enseignement élémentaire pour les plus pauvres et crée des écoles normales dans chaque province. Dans un royaume très décentralisé et où de nombreux particularismes (par exemple linguistiques) demeurent, le projet va même beaucoup plus loin que la loi Guizot puisqu'il prescrit la mise en place d'une école centrale pour les formateurs des écoles normales. En 1838, le marquis de Someruelos présente deux projets de loi sur l'instruction primaire et l'instruction secondaire et supérieure. Seul le premier aboutit et permet au moins d'entériner les mesures de 1836. En 1841, Facundo Infante présente à son tour un plan pour l'enseignement "intermédiaire" et supérieur. En 1845 enfin, le plan d'études général de Pidal organise l'enseignement supérieur selon un système centralisé. Si les similarités avec l'organisation française semblent nombreuses, il en va différemment du côté de l'enseignement. Inspiré notamment des idées britanniques, il accorde par exemple une large place aux sciences ${ }^{121}$. Le retour d'exil des réfugiés libéraux a ainsi suscité un réveil pédagogique et intellectuel en Espagne.

Du côté français, la présence de réfugiés espagnols en France a favorisé la

119 Extrait de la lettre de Guizot aux instituteurs, citée par Bertrand Goujon, Monarchies postrévolutionnaires, 1814-1848, Paris, Seuil, 2012, p. 275-276.

120 Buenaventura Delgado Criado, Historia de la educación en España y América: La educación en la España contemporánea (1789-1975), Madrid, Ediciones Morata, 1994, p. 149-150.

121 Alfonso Capitán Díaz, Breve historia de la educación en España, Madrid, Alianza editorial, 2002, p. 261. 
diffusion de la langue par le biais de l'enseignement des langues vivantes souvent hors du cadre scolaire, ou d'une mode espagnole qui fait parfois la part belle au folklore. Toutefois, cette diffusion relève également d'une stratégie active de la part des réfugiés espagnols qui veulent également initier les ressortissants des pays d'accueil à leur culture. Il y a eu, de la part des réfugiés espagnols, une vraie volonté de mettre en avant leur culture et leur littérature, au-delà des clichés. Les Espagnols en exil sont des nationalistes culturels : leur sentiment identitaire s'exprime d'abord sur le plan culturel et scientifique. Pour le journal El Emigrado observador, l'édition en 1827 d'un Don Quichotte à Paris par le réfugié Joaquín María Ferrer n'est pas seulement un acte littéraire (ou commercial) mais possède une véritable portée patriotique :

"Le Sr. Ferrer n’a rien omis pour que l'édition actuelle du Quichotte en miniature égale celles que les autres nations ont faites de leurs plus célèbres auteurs, et il y est parvenu, à force de travail et de dépenses, en présentant au monde littéraire un tour de force, comme disent les Français. Tout en louant l'idée véritablement patriotique du Sr. Ferrer, nous recommandons la présente édition à tous les hommes de goût raffiné, nationaux ou étrangers, comme étant hautement digne de figurer dans leurs bibliothèques " ${ }^{122}$.

Les intellectuels en exil prennent conscience du décalage entre l'image que les étrangers ont de l'Espagne, un pays attardé, avec son histoire ou ses réalités contemporaines. L'un de leurs autres champs d'action est donc s'atteler à l'écriture de l'histoire de leur pays, pour la jeunesse espagnole comme pour les intellectuels étrangers, à l'exemple de la tâche accomplie par Modesto Lafuente, réfugié christino en France au début des années 1840, avec l'Histoire générale de l'Espagne, publié entre 1850 et 1867, œuvre de référence jusqu'à la Seconde République espagnole ${ }^{123}$.

\author{
Anne Leblay-Kinoshita \\ Archives nationales \\ ac.leblay@gmail.com
}

122 El Emigrado observador, $\mathrm{n}^{\circ}$ 1, juillet 1828, p. 27. Traduction.

123 José Álvarez Junco, L'idée d'Espagne, la difficile construction d'une identité collective au XIX'e siècle, Rennes, Presses universitaires de Rennes, 2011, p. 144-145. 